

\section{REVISTA \\ TEORÍA Y PRÁCTICA \\ DE LA \\ ARQUEOLOGÍA HISTÓRICA LATINOAMERICANA}

ISSN: 2250-866X (impreso) | ISSN: 2591-2801 (en línea)

AÑO VIII, VOLUMEN 9, PRIMAVERA DE 2019

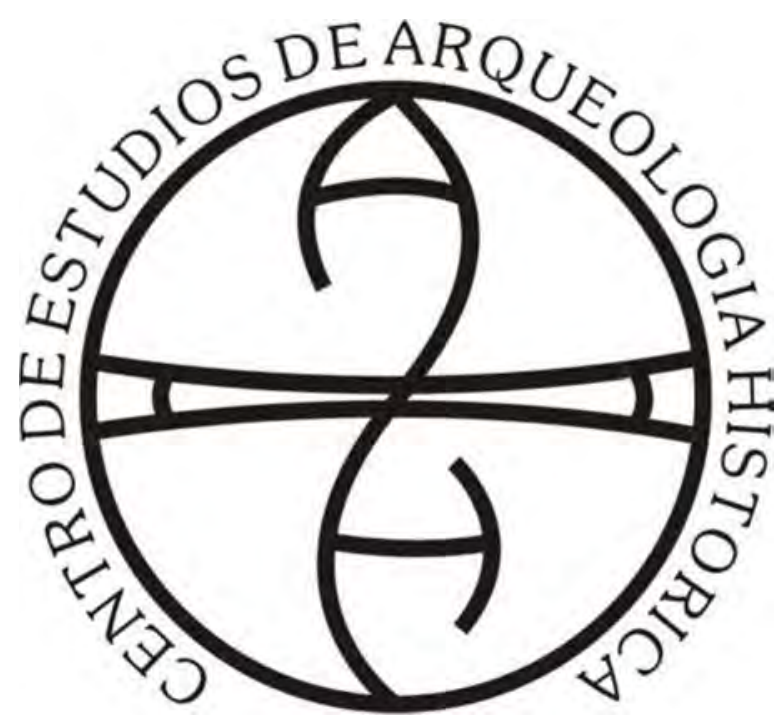

CENTRO de Estudios de ARqueOlOGía HistóricA

FACULTAD DE HUMANIDADES Y ARTES | UNIVERSIDAD NACIONAL DE ROSARIO 
(Universidad Nacional de Rosario, Universidad Nacional de Río Cuarto,

Universidad Nacional de San Juan, Universidad de la República, Universidad Nacional de Trujillo)

\author{
AUTORIDADES DE LA UNIVERSIDAD NACIONAL DE ROSARIO \\ RECTOR: Lic. Franco Bartolacci \\ VICE-RECTOR: Od. Darío Macía \\ SECRETARIO GENERAL: Prof. José Goity \\ SECRETARIA ACADÉMICO Y DE APRENDIZAJE: Dr. Marcelo Vedrovnik \\ SECRETARÍA DE CIENCIA TECNOLOGÍA E INNOVACIÓN \\ PARA EL DESARROLLO: Ing. Guillermo Montero.
}

\author{
AUTORIDADES DE LA FACULTAD DE HUMANIDADES Y ARTES \\ DECANO: Prof. Alejandro Vila \\ VICEDECANA: Prof. Marta Varela \\ SECRETARIA ACADÉMICA: Dra. Marcela Coria
}

\author{
AUTORIDADES DEL CENTRO DE ARQUEOLOGÍA HISTÓRICA \\ DIRECTORA: Dra. Ana Rocchietti \\ SECRETARIA: Prof. Nélida de Grandis \\ PROSECRETARIA: Lic. Marianela Bizcaldi
}

DIRECTORAS - EDITORAS:

Dra. Ana Rocchietti y Prof. Nélida De Grandis

SECRETARIA DE EDICIÓN: Dra. Irene Dosztal

Este número es co-edición de las ponencias

del VIII Congreso Nacional de Arqueología Histórica (2018) entre:

Centro de Estudios en Arqueología Histórica: Directora Ana Rocchietti

Centro de Estudios en Arqueología Regional: Director Fernando Oliva

Centro de Estudios en Arqueología Subacuática: Directora Mónica Valentini

Departamento de Arqueología, Escuela de Antropología: Director Fernando Oliva
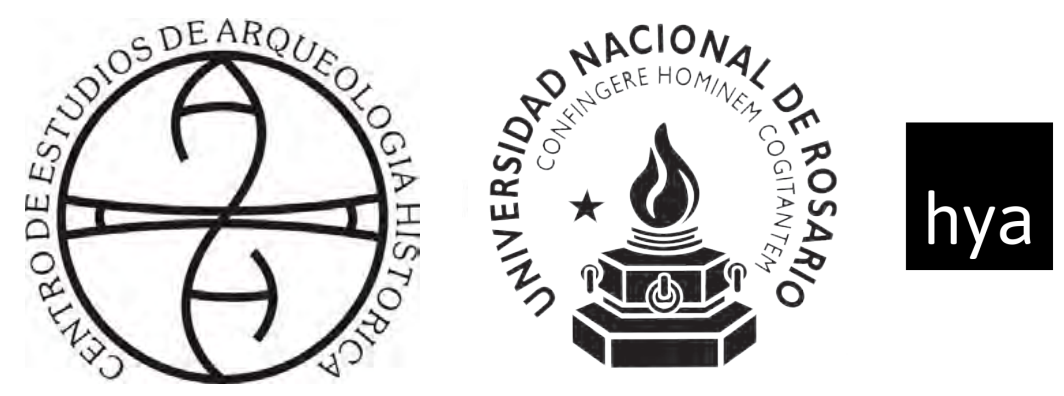

Facultad de Humanidades

y Artes_UNR
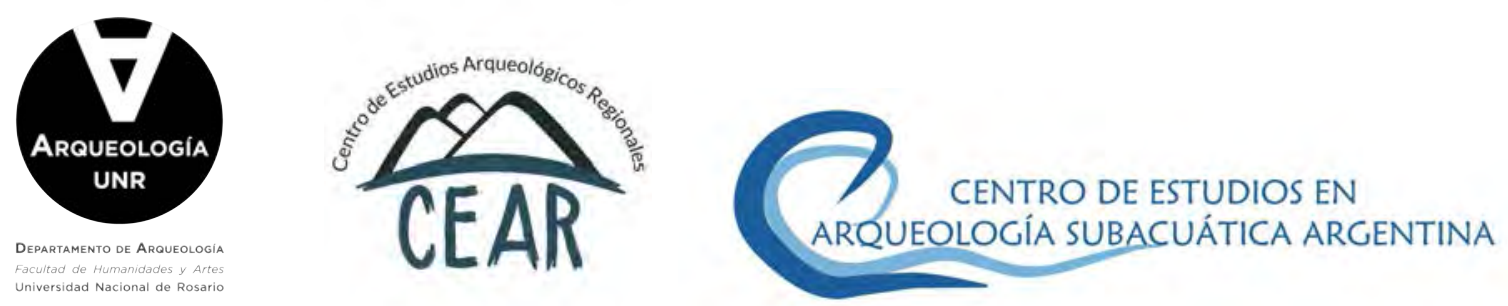
Comité Permanente de los Congresos Nacionales de Arqueología Histórica

Dr. Daniel Schávelzon (Universidad Nacional de

Buenos Aires)

Prof. María Teresa Carrara (Universidad Nacional de Rosario)

Prof. Carlos Baldassarre (Museo Municipal de Río

Grande, Tierra del Fuego) in memoriam

Dr. Mariano Ramos (Universidad Nacional de Luján, CONICET)

Dr. Horacio Chiavazza (Universidad Nacional de Cuyo)

Dra. Ana María Rocchietti (Universidad Nacional de

Rosario, Universidad Nacional de Río Cuarto)

Lic. Facundo Gómez Romero (Universidad Autónoma

de Barcelona)

\section{Comité Científico}

Dra. Tânia Andrade Lima (Universidade Federal do Rio de Janeiro)

Prof. Réginald Auger (CELAT/Département des

Sciences Historiques, Université Laval, Canadá)

Dr. Roberto Bárcena (Universidad Nacional de Cuyo, CONICET)

Dra. Marta Bonaudo (Universidad Nacional de Rosario, CONICET)

Dr. Leonel Cabrera (Universidad de la República, Uruguay)

Dr. Luis María Calvo (Universidad Católica de Santa

$\mathrm{Fe})$

Prof. Juan Castañeda Murga (Universidad Nacional de

Trujillo, Perú)

Dr. Carlos Ceruti (Museo de Ciencias Naturales y

Antropología "Prof. Antonio Serrano”. Paraná)

Dr. Horacio Chiavazza (Universidad Nacional de Cuyo)

Dra. Silvia Cornero (Universidad Nacional de Rosario)

Prof. Pedro Paulo Funari (Universidade Estadual de

Campinas, Brasil)

Lic. Jorge A. Gamboa Velásquez (Universidad Nacional

Santiago Antuñez de Mayolo, Perú)

Dr. Eduardo Alejandro García (Universidad Nacional de

San Juan, CONICET)

Prof. Nélida De Grandis (Universidad Nacional de

Rosario)

Dr. Juan Bautista Leoni (Universidad Nacional de

Rosario, CONICET)

Dra. Amancay Martínez (Universidad Nacional de San

Luis)

Dra. Catalina Teresa Michieli (Universidad Nacional de

San Juan, CONICET)

Lic. Fernando Oliva (Universidad Nacional de Rosario)
Ing. Adrián Pifferetti (Universidad Tecnológica Nacional Regional Rosario)

Dr. Mariano Ramos (Universidad Nacional de Luján, CONICET)

Dra. Ana María Rocchietti (Universidad Nacional de Rosario)

Dr. Daniel Schávelzon, (Universidad Nacional de Buenos Aires, CONICET)

Dra. Carlota Sempé (Universidad Nacional de La Plata)

Dr. Mario Silveira (Universidad Nacional de Buenos

Aires)

Dra. Silvia Simonassi (Universidad Nacional de Rosario)

Dra. Alicia Tapia (Universidad Nacional de Buenos Aires, Universidad Nacional de Luján)

Lic. Mónica P. Valentini (Universidad Nacional de Rosario)

Agrim. Benito Vicioso (Universidad Nacional de Rosario)

\section{Evaluaron este volumen}

Roberto Bárcena, María Teresa Boschin, Leonel Cabrera, Ulises Camino, María Rosa Carbonari, Carlos Ceruti, Horacio Chiavazza, Nicolás Ciarlo, Silvia Cornero, Eduardo Crivelli, Javier García Cano, Martín Gentinetta, María Laura Gili, Carlos Landa, Matilde Lanza, Melina Malandrino, Sebastián Pastor, Victoria Pedrotta, Josefina Piana, Mercedes Podestá, Mariano Ramos, Daniel Schavelzon, Diana Tamburini, Mónica Therrien, Mónica Valentini y María Teresa Boschin

\section{Diseño y diagramación}

Eugenia Reboiro

(eugenia.reboiro@gmail.com)

\section{Curadoría}

Ana Rocchietti e Irene Dosztal

Foto de tapa: Material arqueológico, del texto de Tapia et al.

\section{Propietario responsable:}

Facultad de Humanidades y Artes, Universidad Nacional de Rosario Centro de Estudios de Arqueología Histórica

Entre Ríos 758. Rosario, provincia de Santa

Fe (2000). Argentina.

Telf.: +54 (0341) 4802670

E-mail: ceahunr@gmail.com

Decreto Ley 6422/57 de Publicaciones

Periódicas 


\section{Índice}

Editorial

El origen del patrimonio histórico y su conservación integral en la contemporaneidad.

Yanina Aguilar

Prospección de basurales históricos de la ciudad de Rosario.

Soccorso Volpe y Gustavo Fernetti

Desconstrucción de un paisaje urbano. El Montevideo del extramuros colonial, aportes de la arqueología a su identidad actual. Ana Gamas

La Calota de Ameghino: reconsiderando un viejo tema desde la arqueología histórica.

Daniel Schávelzon

Análisis zooarqueológico de los restos hallados en una estructura sanitaria vinculada a momentos tempranos del actual barrio de Belgrano .71 Mario Silveira, Horacio Padula, Ricardo Orsini y Eva Bernat

La medida del tiempo: el reloj solar jesuita de La Cruz, provincia de Corrientes.

Fernando Oliva y María Cecilia Panizza

Sitio La Quinta: arqueología rural de campos serranos.

Flavio Ribero

Si no hay tabaco que no se note. Prácticas fumatorias en el fortin La Perra (1883-1885), La Pampa.

Alicia Tapia, Virginia Pineau y Melisa Ayelén Auge

El uso del tabaco y las pipas de caolin (clay pipes) en la frontera sur y oeste de la provincia de Buenos Aires en el siglo XIX.. .127

María del Carmen Langiano y Julio Fabián Merlo 
Patrimonio natural, urbano y arquitectónico de la costa en Mar del Plata. Federico Negroni

Huellas urbanas.

Ezequiel Serrot

Paisaje y patrimonio. La industria taninera en el siglo XX.

Provincia de Santa Fe.

Cristina Pasquali, Paola Milicic y Lara Ferré

Los desafíos de las arqueologías históricas latinoamericanas.

Andrés Zarankin

Sellos entre escombrales. Las lozas en el registro urbano

del Área Fundacional de Mendoza (siglo XIX y principios del siglo XX)

Lorena Puebla y Horacio Chiavazza

Evolución edilicia de la Plaza de Mayo de la Ciudad

de Buenos Aires (1580-1853).

Nicolás Ferrino

Gestión patrimonial en el rescate arqueológico y futuro Museo de Sitio

en Moreno 550, Ciudad Autónoma de Buenos Aires, Argentina.

María Eva Bernat, Ricardo Orsini, Horacio Padula y Mario Silvera 


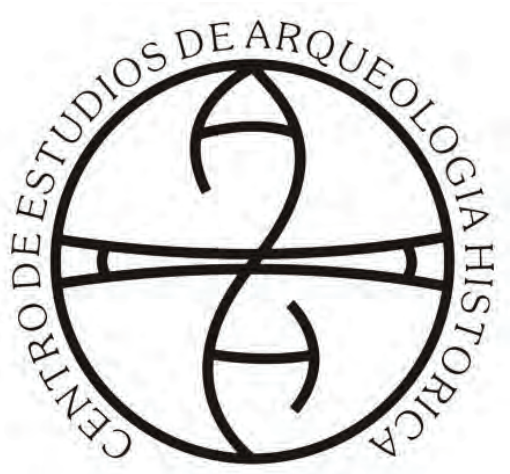

Centro de Estudios de Arqueología Histórica

Universidad Nacional de Rosario
Teoría y Práctica de la Arqueología Histórica

Latinoamericana | Año VIII. Vol. 9 | 2019

Revista del Centro de Estudios de Arqueología

Histórica, Facultad de Humanidades y Artes,

Universidad Nacional de Rosario

https://teoriaypracticaah.unr.edu.ar/index.php/index

ceahunr@gmail.com

ISSN en línea: 2591-2801

ISSN versión impresa: 2250-866X

\title{
EL USO DEL TABACO Y LAS PIPAS DE CAOLIN (CLAY PIPES) EN LA FRONTERA SUR Y OESTE DE LA PROVINCIA DE BUENOS Aires EN EL SIgLO XIX
}

\author{
María del Carmen Langiano* y Julio Fabián Merlo**
}

\begin{abstract}
Resumen
Esta investigación está focalizada en el uso del tabaco y de las pipas de caolín en la frontera Sur y Oeste de la Provincia de Buenos Aires, específicamente en el periodo 1820-1890. Los viajeros, documentos escritos, egodocumentos (Rodino 2003) y partes militares han mencionado el uso del tabaco en ciudades, estancias, pueblos, fuertes y fortines, tanto por parte de los eurocriollos como de las comunidades originarias.

Las pipas de caolín (clay pipes) constan de una cazuela y una caña de paredes delgadas. Y el diámetro de su agujero fue variando a lo largo del tiempo a medida que fue avanzando la producción del tabaco, la tecnología, su uso y reuso de moldes. Estas partes han constituido uno de los elementos diagnósticos para poder precisar la escala temporal de su uso. En cuanto a la escala espacial de este trabajo refiere a los hallazgos de pipas de caolín en contextos arqueológicos de los partidos de Olavarría, Tandil, Coronel Suárez, Azul, Tapalqué, entre otros, El estudio de esta parte de la cultura material ha permitido detectar una diversificación de la cultura material porteña, fenómeno que lentamente se fue extendiendo hacia el área rural y las zonas fronterizas.
\end{abstract}

Palabras clave: tabaco - cultura material - pipas de caolín - sociedad de frontera

\begin{abstract}
This research is focused on the use of tobacco and kaolin pipes in the South and West border of the Province of Buenos Aires, specifically in the period 1820-1890. Travelers, written documents, egodocuments and military parties have mentioned the use of tobacco in cities, ranches, towns, forts and fortlets,

* Langiano María del Carmen INCUAPA-CONICET. Facultad de Ciencias Sociales. Universidad Nacional del Centro de la Provincia de Buenos Aires. Avda. De Valle 5737. Olavarría (B7400JWI). Buenos Aires. Argentina. Tel. (02284) - 450115 mariadelcarmenlangiano@gmail.com.

** Merlo Julio Fabián INCUAPA-CONICET. Facultad de Ciencias Sociales. Universidad Nacional del Centro de la Provincia de Buenos Aires. Avda. De Valle 5737. Olavarría (B7400JWI). Buenos Aires. Argentina. Tel. (02284) - 450115 mariadelcarmenlangiano@gmail.com
\end{abstract}


both by the eurocriollos and the original communities.

The clay pipes consist of a casserole and a thin-walled cane. And the diameter of its hole was changing over time as tobacco production, technology, used and reuse molds progressed. These parts have been one of the diagnostic elements to be able to specify the time scale of its use. Regarding the spatial scale of this work, it refers to the findings of kaolin pipes in archaeological contexts of the parties of Olavarría, Tandil, Coronel Suárez, Azul, Tapalqué, among others. The study of this part of the material culture has allowed detecting a diversification of the material culture of Buenos Aires, a phenomenon that slowly spread to the rural area and the border areas.

Keywords: tobacco - material culture - pipas de caolín - sociedad de frontera

\section{INTRODUCCION}

El consumo del tabaco era usual en América y los primeros europeos observaron cómo los indígenas utiilizaban las hojas de tabaco trituradas, en infusiones, aspirándolas,encendiendo directamente las hojuelas de tabaco o fumándolas en pipas. Este hábito de fumar fue introducido en Inglaterra hacia 1565, por el navegante Hawkins (Volpe 2001). Los encargados de su difusión mundial fueron los marinos holandeses y portugueses, quienes trasladaron la moda a sus diversos puertos de escala, introduciéndola simultáneamente en Persia, Java, Indochina y Japón. A inicios del siglo XVII el tabaco era conocido en todos los continentes, excepto Australia. Esta centuria y el siglo XVIII, fueron los de mayor auge de la industria de pipas europeas, desarrollándose con distintos materiales e infinidad de diseños. De este modo era usual fumar cigarros, usar pipas, aspirar rapé (tabaco en polvo al que se añadía, por ejemplo. mentol, mascar o beber tabaco.

El tabaco fue clasificado por el naturalista Karl von Linné como Nicotiana tabacum, en honor al literato y diplomático francés Jean Nicot de Villemain, quien fue el primero en llevarlo a Francia desde Portugal en 1560 (Figura 1). Como consecuencia, Francia se sumó también como país productor de pipas, manufacturas fueron rápidamente exportadas a las colonias, donde inmigrantes europeos, criollos y esclavos africanos demandaban estos productos. Así, se diversificó la importación de pipas de fumar y la elaboración manual de las mismas por parte de artesanos locales (Figura 2). Ejemplo de esta producción son las frágiles y livianas pipas de caolín (clay pipes) y las de terracota, "generalmente atribuidas a contextos prehispánicos y en otros a contextos de contacto” (Zorzi y Davey 2011:203) o de filiación africana (Schávelzon 2003) que han sido recuperadas en la ciudad de Buenos Aires. 
Teoría y Práctica de la Arqueología Histórica Latinoamericana | Año VIII. Vol. 9 | 2019

ISSN en línea: 2591-2801 | ISSN versión impresa: 2250-866X
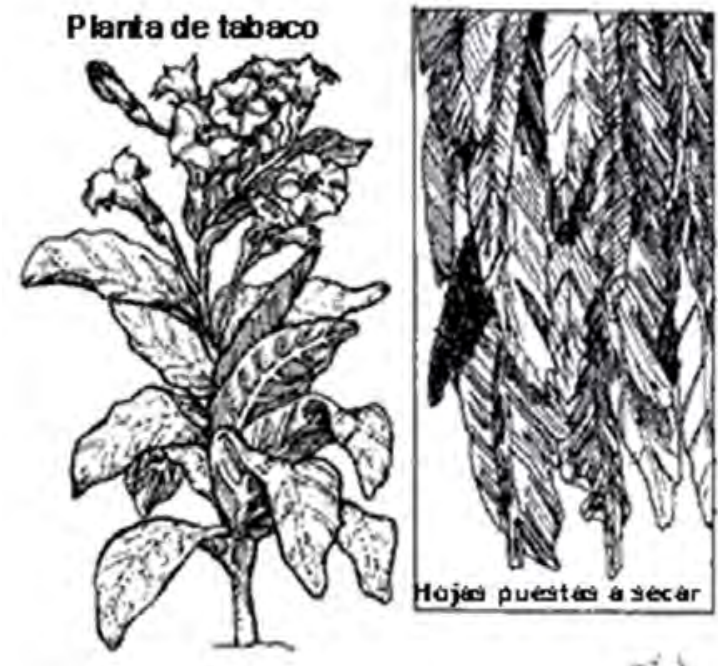

Figura 1. Nicotiana tabacum.

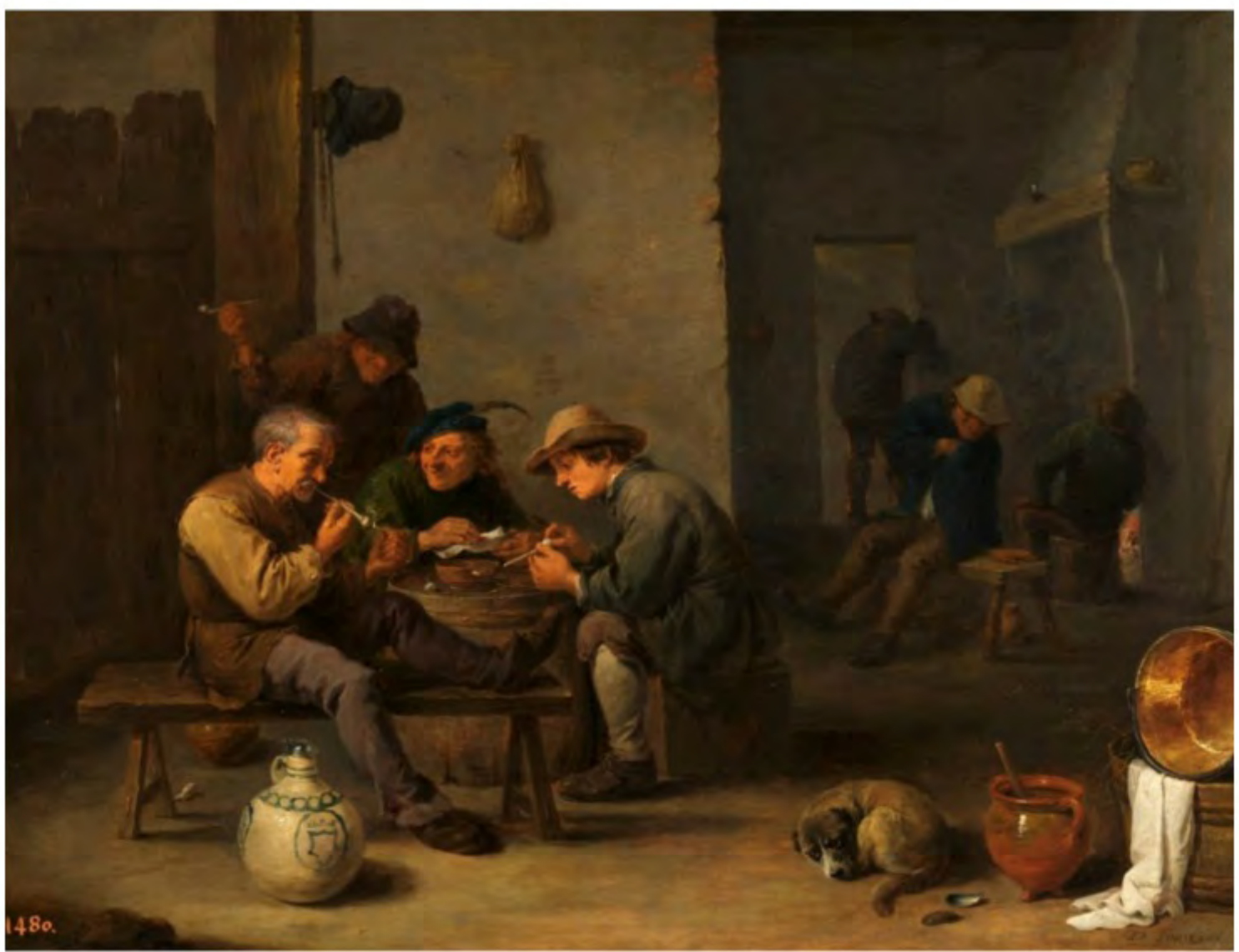

Figura 2. Fumadores de pipa, óleo de mediados del siglo XVIII. 
Las primeras pipas europeas fueron confeccionadas con arcilla (clay pipes) y su principal componente era el caolín (espuma de mar que es el nombre común dado a este material de roca porosa de origen volcánico, el hidrosilicato de magnesio). inglaterra fue su primer productor a partir del siglo XVI, en principio en forma protoindustrial y luego utilizando moldes. Holanda comenzó a elaborar pipas a principios del siglo XVII, al ser introducida por pipermakers ingleses que huyeron de una persecución religiosa. de Jaime I, sucesor de Isabel I. Uno de estos exiliados fue William Baernelts, que adoptó el nombre holandés de Willem Barentz y abrió una manufactura en 1617 en Gouda (Walker 1976). Francia se sumó también como país productor de pipas que fueron rápidamente exportadas a las colonias, donde inmigrantes europeos, criollos y esclavos africanos demandaban estos productos. Así, se diversificó la importación de pipas de fumar y la elaboración manual de las mismas por parte de artesanos locales. Ejemplo de esta producción son las frágiles y livianas pipas de caolín (clay pipes) y las de terracota (Figura 3), "generalmente atribuidas a contextos prehispánicos y en otros a contextos de contacto” (Zorzi y Davey 2011:203) o de filiación africana (Schávelzon 2003) que han sido recuperadas en la ciudad de Buenos Aires.

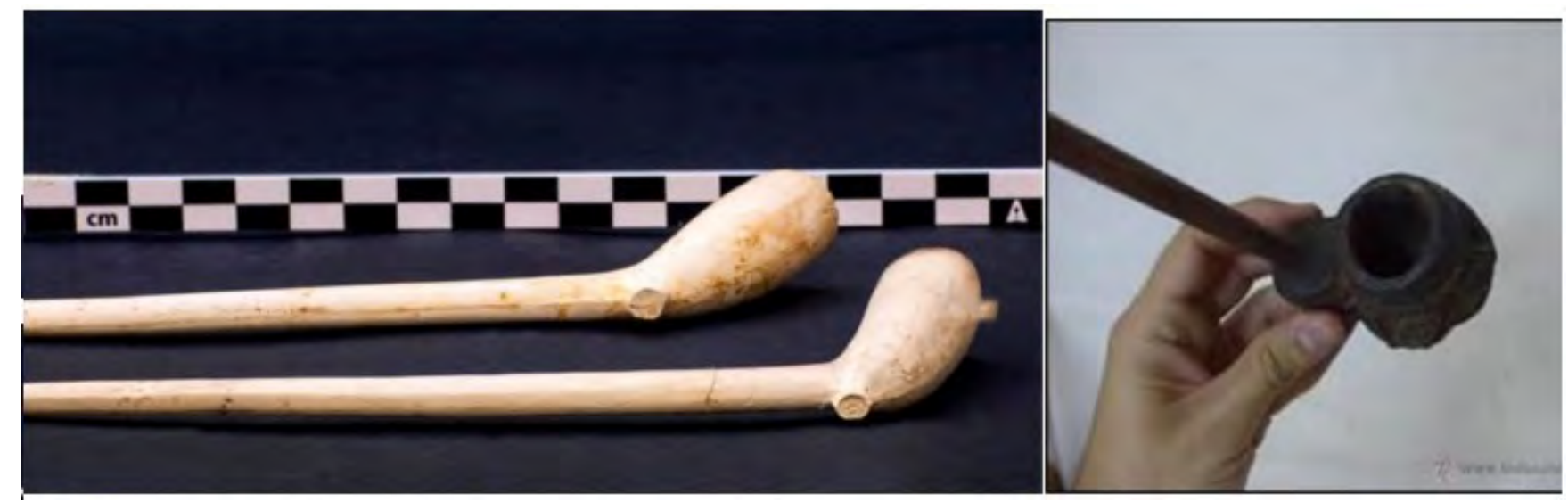

Figura 3. Ejemplos de pipas de caolín (izquierda) y de terracota (derecha).

Las pipas de caolín (clay pipes) constan de una cazuela y una caña de paredes delgadas; el diámetro de su agujero fue variando a lo largo del tiempo a medida que fue avanzando la producción del tabaco, la tecnología, el uso y el reuso de los moldes. En principio esto ha constituido un elemento diagnóstico para poder precisar la escala temporal de su fabricación u utilización. La producción del tabaco de pipa se fue desarrollando durante el siglo XVII, se fue modificando la caña o tubo, el diámetro de su orificio y del recipiente del hornillo o cazoleta.

Las tipologías morfológicas y comerciales permiten datar a las pipas de caolín, pues los comerciantes crearon modelos exclusivos y marcaron sus inscripciones en ellas. Su desarrollo inicial en muchos centros locales en todo el Reino Unido y en muchas partes de los Países Bajos permitió una distribución limitada dentro de su área de producción, excepto en los casos de las ciudades portuarias y centros comerciales en el extranjero. Estas primeras pipas tenían como rasgo típico un corto vástago cuyo orificio tenía un gran diámetro y una pequeña "bellota” en forma de tazón ruleteado (rouletted) en un ángulo de la cara, lejos del fumador. A medida que la producción del tabaco de pipa se fue desarrollando durante el siglo XVII, la caña o tubo se hizo más larga, el tamaño de su orificio fue variando como consecuencia del reuso de los moldes y el recipiente del hornillo o cazoleta era más grande. A principios del siglo XVIII, 
esta última adoptó una forma de lados más rectos y grandes, sin ruleteado alrededor del borde y con el hornillo perpendicular al tubo (Walker 1976).

Se encuentran marcas de fabricación en los tubos o cañas tanto durante los siglos XVII como XVIII. Estas se dividen en dos categorías principales: marcas de relieve en el tubo estampadas ya sea con un dado o incorporadas en el molde de la pipa y marcas estampadas que forman una impresión negativa en la caña y fueron hechas con un troquel o se aplican por un dispositivo similar a través del cual se enrolla el vástago.

Como regla general, las cañas de pipas inglesas y holandesas de la primera mitad del siglo XVII fueron estampadas sobre todo en el talón. A partir de finales del siglo XVII, hasta el tercer cuarto del siglo XVIII las marcas más comunes tienen forma de un cartucho moldeado en el lado derecho o izquierdo del hornillo, con iniciales estampadas en los lados del talón o como un nombre abreviado impreso en la espuela.

Las pipas holandesas de los siglos XVII y XVIII se distinguen de las inglesas, no sólo por su forma de la cazoleta sino también por la presencia de ruleteado alrededor del borde y porque los pipemakers continuaron estampando sus pipas en el talón, a menudo utilizando marcas minúsculas. El decorado de las pipas holandesas de la primera mitad del siglo XVIII era muy sencillo, simplemente alguna corona o símbolo, lisas en muchos casos o con pequeñas estrías o cantos finamente ruleteados. Afirma Leclaire (2008) que la ciudad de Gouda, al sur de Holanda, fue un importante centro productor de pipas regulares, finas y "porcelanas", elaboradas con el sistema de arrollado a mano (hand rolled clays). Entre los años 1700-1760 eran muy refinadas y tenían motivos decorativos, las más grabadas aparecen entre 1735 a 1755, cuando la familia de plateros Van Oye se estableció en Gouda. Las de tamaño medio eran muy populares entre la clase media y las pequeñas entre la clase menos favorecida de la sociedad. Es oportuno señalar que los fabricantes holandeses se identificaban con sus propias marcas, por ejemplo la tabakscomfoor (1708-1846) o la Kofflepor (1720-1725).

Las pipas de arcilla dispersan naturalmente el calor y la cazoleta se calienta rápidamente por el proceso. Las fábricas alemanas de Hor revolucionaron la industria a finales del siglo XVII al desarrollar dos piezas: cazoleta de arcilla y boquilla removible (de cerezo u otro material), de este modo la quilla permanece fría, por eso eran populares las boquillas largas. El tamaño de la cazoleta se fue haciendo más grande a medida que el precio del tabaco bajaba; entre el precio y el tamaño había una relación inversamente proporcional. De acuerdo a las investigaciones efectuadas por Hernández Godoy y Arrazcaeta Delgado (2009), las pipas protoindustriales fueron hechas mediante un complejo proceso de preparación de la arcilla, modelado, conformación de su forma final en un molde metálico de dos partes, eliminación de las rebabas o sobrantes y secado natural. Después eran introducidas en moldes de tierras refractarias y cocidas en hornos cerrados en una temperatura controlada de 200 a $300^{\circ} \mathrm{C}$ durante 16 a 20 horas. El bruñido se llevó a cabo en todas las épocas, aunque es más común en las pipas de Londres del siglo XVII. La superficie de la pipa terminada era pulida con una pequeña herramienta, mediante una serie de trazos verticales paralelos recorriendo todo el recipiente y, por lo general, también a lo largo de la longitud de la caña. Este proceso cuidadoso de las pipas incidía en su calidad, registrándose piezas muy buenas, buenas, regulares o malas.

El diámetro del conducto fue variando a lo largo del tiempo Dada su extrema fragilidad, se estima que una pipa de caolín tiene una vida útil promedio de no más de dos años, lo que constituye un importante indicador cronológico. La cazoleta, también denominada hornillo o tabaquera, donde se coloca el tabaco, puede presentar un tacón o talón para su apoyo (cuando éste es algo más largo se denomina espuela o pedúnculo). La caña o tubo, por donde se inhala el humo del tabaco de la cazoleta es hue- 
Teoría y Práctica de la Arqueología Histórica Latinoamericana | Año VIII. Vol. 9 | 2019

ISSN en línea: 2591-2801 | ISSN versión impresa: 2250-866X

ca y su largo puede variar. La boquilla, constituye la parte distal de la caña y en ella se introduce la boca. Las pipas con boquillas cortas, en la literatura también son nombradas “cazoletas sin tubo” (Schávelzon 2000:186), a las que se les agregaba un aditamento como cañas huecas, que facilitaban la inhalación del humo y evitaban las quemaduras en las manos. Las pipas de caolín de caña alargada, son distinguidas por su cazoleta blanca con una extensa caña que podía sobrepasar los $80 \mathrm{~cm}$, aunque fueron más comunes las de 30 a $25 \mathrm{~cm}$, cuyo objetivo era extender su vida útil y facilitar que el humo llegara frío a la boca (Schávelzon 2000: 239). Mediante el estudio de la forma, tamaño y características de la cazoleta de una pipa es posible determinar un rango de fechas, y tal vez el origen regional para ese ejemplar particular.

La cazoleta, también denominada hornillo o tabaquera, donde se coloca el tabaco, puede presentar un tacón o talón para su apoyo, y cuando éste es algo más largo se denomina espuela o pedúnculo. La caña o tubo, que es hueca y por donde se inhala el humo del tabaco de la cazoleta, puede ser corta o muy larga. La boquilla, constituye la parte distal de la caña y en ella se introduce la boca. A su vez, las pipas con boquillas cortas, en la literatura también son nombradas "cazoletas sin tubo" (Atkinson y Oswald 1972; Schávelzon 2000:186), a las que se les agregaba un aditamento como cañas huecas, que facilitaban la inhalación del humo y evitaban las quemaduras en las manos. También son conocidas las pipas de caolín de caña alargada, las distingue su cazoleta blanca con una extensa caña que podía sobrepasar los $80 \mathrm{~cm}$, aunque fueron más comunes las de 30 a $25 \mathrm{~cm}$, cuyo objetivo era mantenerlas en uso, aunque éste se fuera gastando por el contacto con la boca y así facilitar que el humo llegara frío a la boca (Schávelzon 2000:239).

\section{DOCUMENTOS ESCRITOS, EGODOCUMENTOS, FOTOGRAFÍAS, POSTALESY PINTURAS, SIGLO XIX}

Esta investigación está focalizada en el uso del tabaco y de las pipas de caolín en la Frontera Sur y Oeste de la provincia de Buenos Aires, específicamente en el periodo 1820-1890. Los viajeros, documentos escritos, egodocumentos y partes militares han mencionado el uso del tabaco en ciudades, estancias, pueblos, fuertes y fortines, tanto por parte de los eurocriollos como de las comunidades originarias (Langiano 2015).

A continuación podemos leer cómo María Martrín Donos Camus, quien vivía en la Estancia La Tigra en el partido de Olavarría, establecimiento ganadero cercano al Fortín El Perdido describe en 1885 la llegada de jefes y guerreros indígenas y su gusto por el tabaco.

"Les femmes mettaient pied á terre avec quelques hommes, dessellaient les chevaux, tiraient de dessous le recado... Les demmes indiennes qui sont de véritable betes de sommes, montaient les tentes, pusi quand les hommes avaient abbattus les moutons qui les attendaient, allumaient les feux, preparaient la nmpurriture tandis que les chef et les guerriers favoris survellaient du haut de leur cheval en fumant leur pipe que tout fut pret. Si une dispute s'elevait entre les femmes ou les enfants, un d'eux se mobilisait et de quelques coups de rebenque bien appliqué faisait tout renrer dans l'ordre...." (María Martrin Donos Camus, 1885 Ms).

"Las mujeres pusieron pie a tierra como cualquier hombre, desensillaron los caballos, se tiraban encima del recado... Las mujeres indias, que son verdaderas bestias de carga levantaban los toldos y cuando los hombres habían carneados los corderos que les correspondían, encendían el fuego y preparaban la comida mientras que el jefe y los guerreros favoritos vigilaban desde arriba de sus caballos, fumando sus pipas hasta que todo estuviera listo. Si entre las mujeres o los chicos surgía 
alguna disputa, uno de ellos se movilizaba y con unos rebencazos bien aplicados, restablecía el orden...”. (María Martrin Donos Camúa 1885-1907 Ms).

Consultado un documento del Archivo del Juzgado de Paz de Saladillo de 1878 hace referencia a la entrega de tabaco y papel a las guarniciones de los fortines de la frontera Sur:

“Al Capitán Comandante de la fuerza del Saladillo

Dn. Dionisio Pereyra

Por el Cabo Pablo Escudero remito á ud docientas veinte y seis libros para setenta y cuatro varas tabaco, setenta y cuatro cuadernillos papel, ciento cincuenta y ocho panes de jabón y treinta y siete libros del para racionarla fuerza de ese punto que son 35 plazas y las del Fortín Arevalo 33 plazas- á razón de 3 libras yerba, una del tavaco, un cuadernillo papel, 2 panes jabón y media libra sal por cada uno, las que remitirá ud. al fortín, dejando las de ese punto.

Dios guíe a ud. sus años

Juan A Noguera” (Archivo del Juzgado de Paz de Saladillo circa 1870).

A continuación se presentan imágenes de pinturas, postales (Pezzimenti 2002) y fotografías de mediados y finales del siglo XIX y principios del XX, de la época mostrando a indígenas y peones fumando (Figuras 4, 5 y 6).

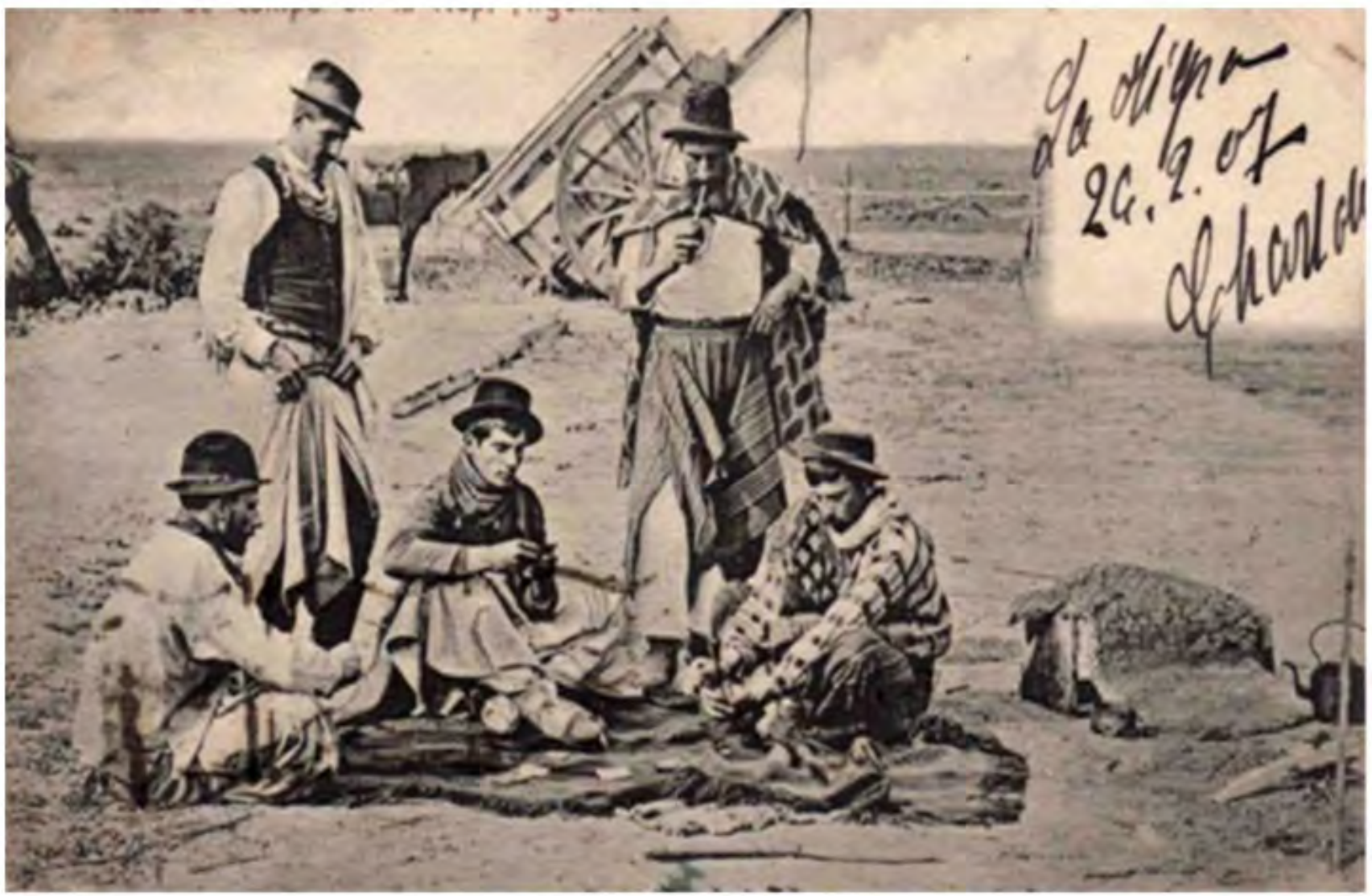

Figura 4. Postal del 29 de enero de 1907 mostrando a peones de campo fumando (Gentileza Eduardo Bernaudo). 
Teoría y Práctica de la Arqueología Histórica Latinoamericana | Año VIII. Vol. 9 | 2019

ISSN en línea: 2591-2801 | ISSN versión impresa: 2250-866X

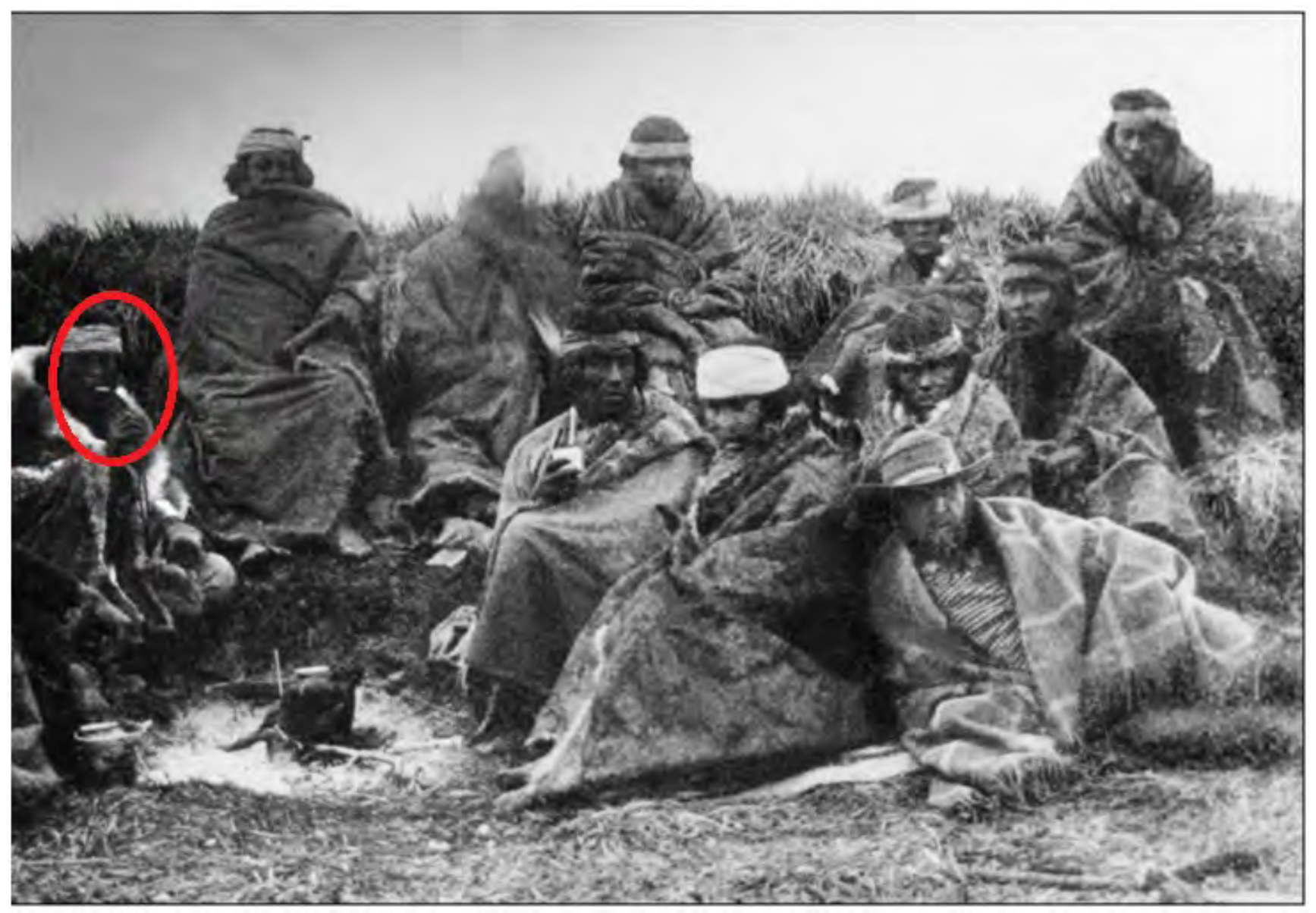

Figura 5. Fotografía tomada por el fotógrafo oficial de Roca en su "Campaña al desierto".

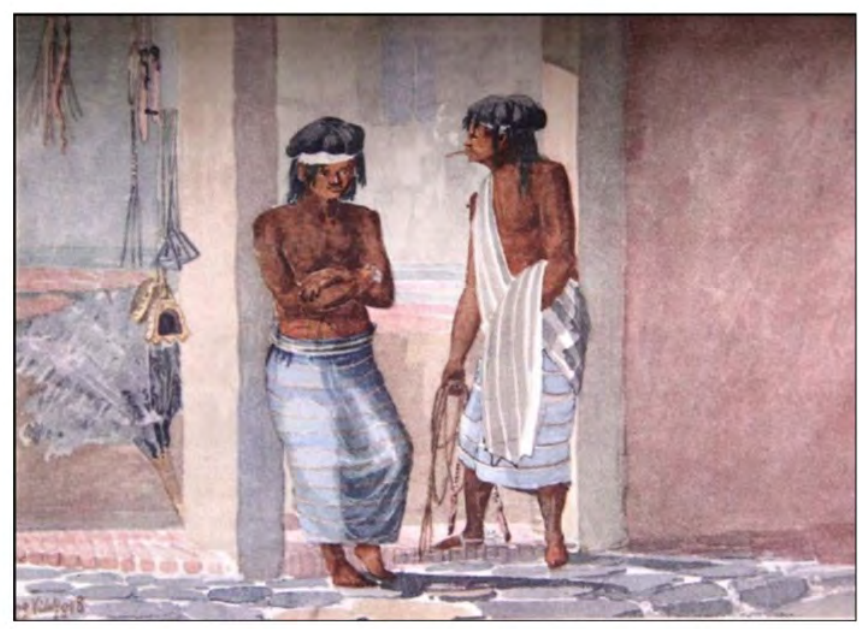

Figura 6. Acuarela de Morel (mediados del siglo XIX). 


\section{PIPAS DE CAOLIN RECUPERADAS EN SITIODS ARQUEOLOGICOS}

En la Figura 7 podemos observar la escala espacial de esta investigación que refiere a los hallazgos de pipas de caolín en contextos arqueológicos de Olavarría, Tandil, Coronel Suárez, Azul, Tapalqué, entre otros, partidos de la provincia de Buenos Aires.

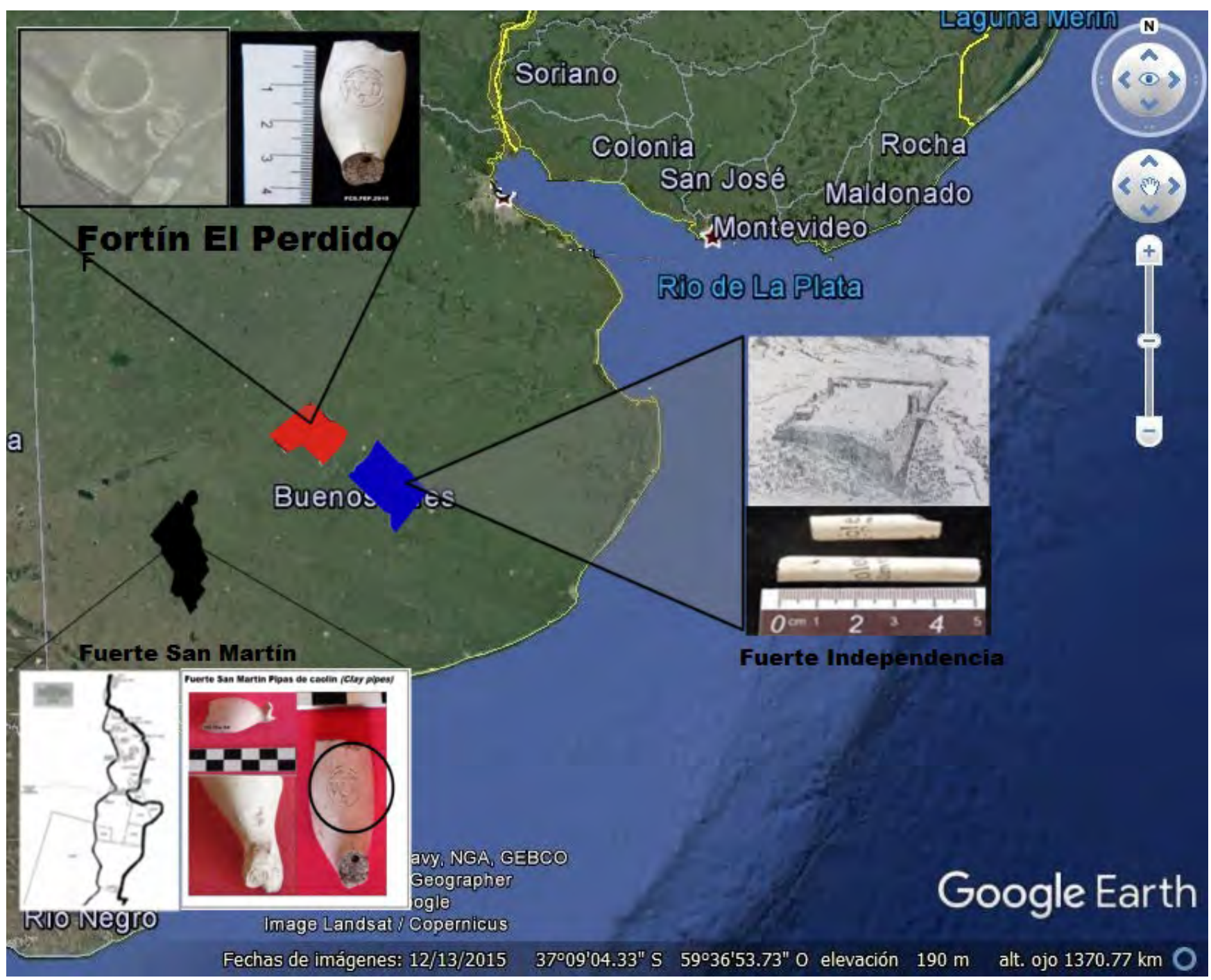

Figura 7. Ubicación de los sitios de la Frontera Sur: Fuerte Independencia 1823, Fortín El Perdido 1865 y Fuerte San Martín 1872.

Se han informado pocos hallazgos arqueológicos de pipas de fumar de arcilla blanca o de caolín en nuestra área de estudio, la campaña y el ámbito fronterizo; sin embargo no sucede lo mismo en zonas urbanas. Como antecedentes en zonas de fortines , podemos citar excavaciones en el Fortín Miñaña (Gómez Romero 1999) y en el Cantón Tapalqué y en el casco urbano de Azul (Mugueta 2011; 2013), en el área urbana las de Rosario (Volpe 2001), las de Bolívar 373 (Zorzi y Davey 2011), Plaza Roberto Arlt y casa Ezcurra (Schávelzon 2003), entre otras, en Capital Federal. 
Se encuentran marcas de fabricación en los tubos o cañas tanto durante los siglos XVII como XVIII. Estas se dividen en dos categorías principales: marcas de relieve en el tubo estampadas ya sea con un dado o incorporadas en el molde de la pipa y marcas estampadas que forman una impresión negativa en la caña y fueron hechas con un troquel o se aplican por un dispositivo similar a través del cual se enrolla el vástago. Se encuentran marcas de fabricación en los tubos o cañas tanto durante los siglos XVII como XVIII. Estas se dividen en dos categorías principales: marcas de relieve en el tubo estampadas ya sea con un dado o incorporadas en el molde de la pipa y marcas estampadas que forman una impresión negativa en la caña y fueron hechas con un troquel o se aplican por un dispositivo similar a través del cual se enrolla el vástago. Para clasificar tipológicamente las pipas de caolín recuperadas en esta zona de la frontera Sur se contemplaron las siguientes variables: presencia / ausencia de marcas, letras o sellos y atributos de las distintas partes de la pipa como diámetros del tubo y del conducto, medidas del hornillo o cazuela y presencia de ruleteado (rouletting).

El material analizado hasta el momento proviene de la Localidad arqueológica El Perdido, donde se recuperaron dos fragmentos de hornillo; el Fuerte San Martín, donde se obtuvo un fragmento de una caña o tubo, en buen estado de conservación pero fragmentados en muy variada proporción y en el Fuerte Independencia, donde se recuperaron dos tubos con inscripciones en francés (Tabla 1). En el caso de la Localidad Arqueológica El Perdido, fueron recuperados en el sitio Tapera 1 en tareas de recolección superficial sistemática (transectas 1 y 5 Norte), en el Fuerte San Martín en recolecciones superficiales (transecta d 9 NE e.) y en el Independencia en las excavaciones efectuadas en el patio de la Iglesia Danesa, sector donde se ubicaba la entrada a la fortificación.

Tabla 1. Pipas de caolín (Clay pipes) recuperadas en la Localidad arqueológica El Perdido, Fuerte San Martín y Fuerte Independencia.

\begin{tabular}{|c|c|c|c|c|c|c|}
\hline $\mathbf{N}^{0}$ inventario & parte de la pipa & Procedencia & Observaciones & Tubo & Conducto & cronologia aprox. \\
\hline \multicolumn{7}{|c|}{ LOCALIDAD ARQUEOLÓGICA EL PERDIDO } \\
\hline 2160 & caña o tubo & \begin{tabular}{|c|} 
RS T1 MS N \\
Tapera I \\
este del fortin
\end{tabular} & Sección circular & $7 \mathrm{~mm}$ & $\begin{array}{l}\text { dámetro externo } 2 \mathrm{~mm} \\
\text { diámetro interno } 1,3 \mathrm{~mm}\end{array}$ & Siglo XIX \\
\hline 2910 & $\begin{array}{c}\text { fragmento } \\
\text { del } \\
\text { hornillo }\end{array}$ & \begin{tabular}{|c|} 
RS T5 MS N \\
Tapera I \\
al E del fortin
\end{tabular} & $\begin{array}{c}\text { Terminado. Sello } \\
\text { con hoja de } \\
\text { tabaco y letras W D } 7\end{array}$ & & & Siglo XIX \\
\hline \multicolumn{7}{|c|}{ MNE $=2$} \\
\hline \multicolumn{7}{|c|}{ FUERTE SAN MARTIN } \\
\hline $\begin{array}{c}70 \\
\text { MNE }=1\end{array}$ & $\begin{array}{l}\text { Frag. De Hornillo } \\
\text { o cazoleta }\end{array}$ & $\begin{array}{l}\text { RST D9 NE } \\
\text { Barranca } \\
\text { de arroyo } \\
\end{array}$ & Terminado. & & & Siglo XVIII - XIX \\
\hline \multicolumn{7}{|c|}{ MNE $=3$ ( 2 cazoletas y un tubo $)$} \\
\hline \multicolumn{7}{|c|}{ FUERTE INDEPENDENCIA } \\
\hline 54 & caña o tubo & C. 1. N.I & Sección circular & $7 \mathrm{~mm}$ & $\begin{array}{l}\text { diámetro extermo } 2 \mathrm{~mm} \\
\text { diámetro interno } 1,3 \mathrm{~mm}\end{array}$ & Siglo XIX \\
\hline 155 & caña o tubo & C. 1, N:II & Sección circular & $7 \mathrm{~mm}$ & $\begin{array}{l}\text { diámetro extermo } 2 \mathrm{~mm} \\
\text { diámetro interno } 1,3 \mathrm{~mm}\end{array}$ & Siglo XIX \\
\hline
\end{tabular}


La pieza FCS FEP 2910 es un fragmento de cazoleta con espuela (spur) de $34 \mathrm{~mm}$ de alto por 21 mm de diámetro, su cámara de combustión sería aproximadamente de unos $40 \mathrm{~mm}$ de profundidad. La base del hornillo se encuentra fragmentada por lo que no se puede determinar la presencia del talón plano o espolón que le permite apoyarla y donde generalmente se encuentra la marca del comerciante. El hornillo mide aproximadamente $25 \mathrm{~mm}$ de boca por $40 \mathrm{~mm}$ de largo. Se observa un sello circular de $8 \mathrm{~mm}$ impreso con el dibujo de una hoja de tabaco y la inscripción WD 7 en la parte de la cara al fumador (Figura 8). Según Leclaire (2008) es una pipa de tabaco que se produjo en el sur de Francia, se puede fechar en el siglo XIX (circa 1820-1880). El largo de la pipa original es de 16,5 cm.

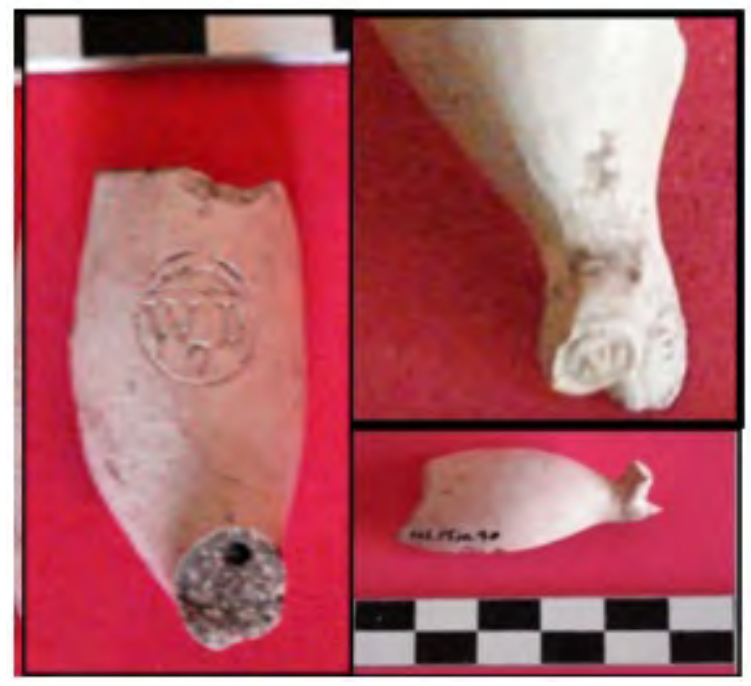

Figura 8. Pipa de caolín recuperada en el Fortín El Perdido.

El ítem FCS FSM 70 es parte de un hornillo blanco en forma de cono ligeramente inclinado hacia adelante, de paredes delgadas y frágiles. Su cámara de combustión es de unos $40 \mathrm{~mm}$ de profundidad. Como en el caso de la pieza FCS FEP 2910 podría ser montada en un vaciado para poder reconstruir la forma de la pipa original. La pipa se ve, en principio, como un producto holandés que dataría de 1750 a 1790. El espolón en la base de la cazuela se ve holandesa por su forma, el estilo y las marcas en zigzag en el talón y la cazuela. Tampoco se puede descartar a Suecia o la zona del Mar Báltico, dado que en principio se realizaron copias renanas del estilo holandés. Las marcas holandeses se siguieron utilizaron a lo largo de varias generaciones, durante un siglo o más. en el caso del fragmento recuperado en el Fuerte San Martín aparece el sello circular con el número 17 sin corona que, de acuerdo al Catálogo de Gouda Clay Marks, podría corresponder al período 1727-1865.

Durante las excavaciones sistemáticas realizadas en la Iglesia Danesa, situada en la ciudad de Tandil se recuperaron dos fragmentos del tubo de las pipas, con la inscripción "Fiolet S'Omer", "FIOLET AS -0 mar”, ambos fragmentos son de origen francés (ver Figura 9). 


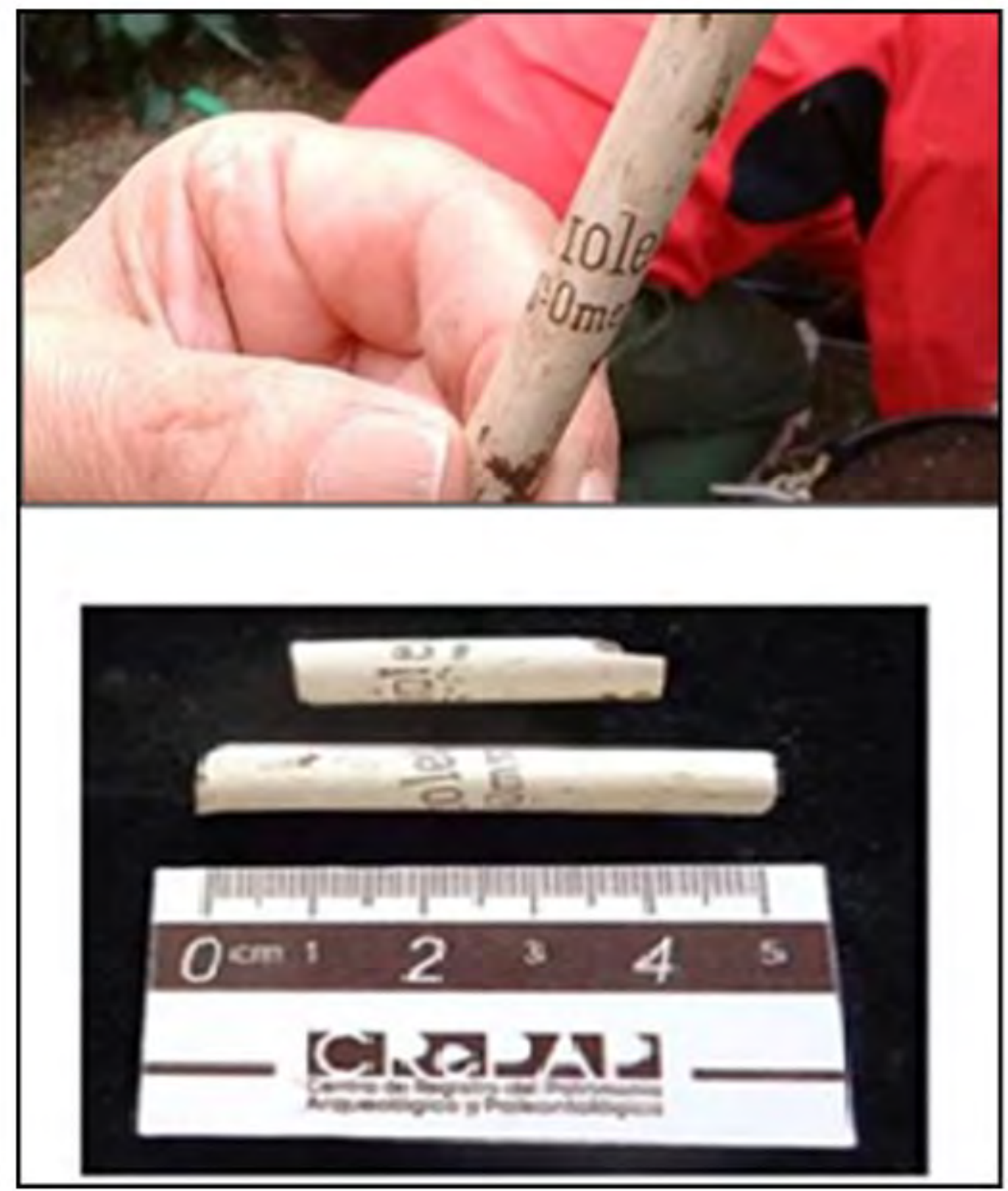

Figura 9. Pipas de caolín recuperadas de la Iglesia Danesa de Tandil.

Analizados otros sitios con hallazgos arqueológicos de pipas de caolín pudimos observar ejemplos similares en el Fuerte General Paz (Leoni et al. 2007), allí se recuperaron fragmentos de pipas de caolín de Glassgow Escocia y la francesa Friolet (Figura 10). 
Teoría y Práctica de la Arqueología Histórica Latinoamericana | Año VIII. Vol. 9 | 2019

ISSN en línea: 2591-2801 | ISSN versión impresa: 2250-866X

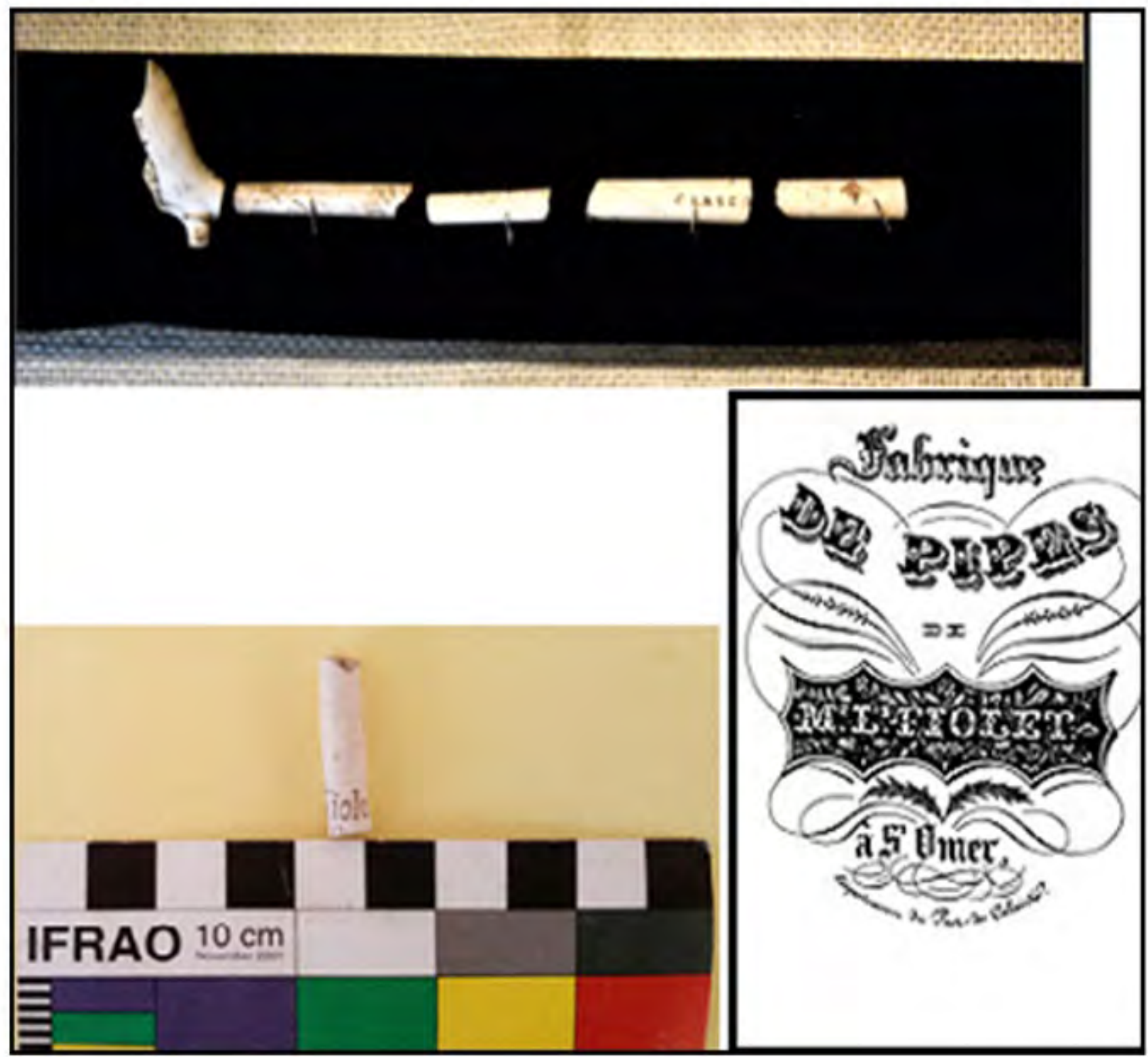

Figura 10. Fragmentos recuperados en el Fuerte General Paz (fotos gentileza Diana Tamburini).

Del mismo modo, en sitios cercanos al Fuerte San Serapio Mártir y en casco urbano de Azul se recuperaron pipas de caolín similares.

\section{CONSIDERACIONES FINALES}

El contexto de hallazgo de las piezas recuperada en asociación con lozas impresas y vidrios moldeados, sugiere una datación aproximada de mediados del siglo XVIII y fines del siglo XIX.

Esa cronología es acorde con el tamaño de la cazoleta, característica que suele ser usada como indi- 
cador de una cronología tardía, en la que el precio del tabaco permitía o no, cargar las pipas más abundantemente.

Durante el siglo XIX en época a la consolidación del Estado Nacional, se afirmó el proceso de inserción del país en un sistema económico a escala global. Esto condujo a un flujo comercial constante de todo tipo de productos que llegaban de zonas muy distantes (Rapoport 1988). Durante esos años se masificó la llegada al país, y especialmente al puerto de Buenos Aires, de inmigrantes europeos (Devoto 2003), quienes traían consigo no sólo costumbres como fumar sino utensilios personales como las pipas de caolín. Todas estas circunstancias conllevaron en principio a una notable diversificación de la cultura material porteña (Schávelzon 1991). Este fenómeno poco a poco va extendiéndose, llegando al área rural ya las zonas de frontera esto se puede apreciar en hallazgos arqueológicos. El estudio de esta parte de la cultura material ha permitido detectar una diversificación de la cultura material porteña, fenómeno que lentamente se fue extendiendo hacia el área rural y las zonas fronterizas.

Es oportuno aclarar que la dificultad que ha implicado determinar con precisión el área de manufactura de las piezas se la ha considerado como un estímulo para dar a conocer más sobre los diversos centros de producción, tecnologías, formas de distribución y variedad de estilos de los materiales presentes en la Frontera Sur.

De acuerdo a lo que puede apreciarse en los contextos arqueológicos porteños, durante el siglo XIX las pipas de caolín fabricadas en Inglaterra eran las más frecuentes (Schávelzon 1991), hecho que no sorprende si se tiene en cuenta el caudal de manufacturas inglesas que arribaron al puerto de Buenos Aires durante la segunda mitad de dicha centuria. Por el contrario y, de acuerdo al estado actual de conocimiento sobre las pipas de caolín en la campaña, si bien en general es muy fragmentario, en particular en lo que refiere a los sitios estudiados (Fuerte San Martín, Localidad arqueológica El Perdido y El Fuerte Independencia), las pipas son de procedencia holandesa y francesa. Cabe aclarar que se han recuperado fragmentos de pipas francesas similares, pero manufacturadas en St. Omer (Francia) y en Glasgow (Escocia) en el Fortín Miñana (Gómez Romero 1999). En cuanto a los estudios realizados por Mugueta (2011 y 2013) en el Cantón Tapalqué y en los sitios Las Flores 580 y Bolívar 420 en Azul, en el primer caso, se menciona su aparición pero no se han publicado resultados. En un sector cercano al Fuerte San Serapio Mártir recupera seis pipas de caolín de procedencia inglesa y en el casco urbano otras con figuras chinescas en su hornillo, asociadas a lozas y mayólicas de fines del 1700.

\section{Agradecimientos}

Al Instituto de Investigaciones INCUAPA - CONICET - UNICEN dirigido por el Dr.G Politis y el Lic. J. L. Prado). A las municipalidades de Olavarría, Coronel Suárez , Nueve de Julio, General Alvear y Tandil. A la comunidad de la Iglesia Danesa de Tandil; ,A colegas y estudiantes de la Facultad de Ciencias Sociales de Olavarría UNICEN.

\section{REFERENCIAS CITADAS}

\section{Atkinson, D. R. and A. Oswald, A.}

1972. A Brief Guide for the Identification of Dutch Clay Tobacco Pipes Found in England. Post Medieval Archaeology 6 (1972): 17-182.

\section{Catálogo Gouda Clay Marks}

http://www.goudapipes.nl/\&prev=search 


\section{Devoto, $\mathbf{F}$.}

2003. Historia de la inmigración argentina. Ediorial Sudamericana, Buenos Aires.

\section{Gómez Romero, $\mathbf{F}$.}

1999. Sobre lo arado el pasado Arqueología Histórica en los alrededores del Fortín Miñana (18601869). Editorial Biblos, Azul.

\section{Hernández Godoy. S. T. y R. Arrazcaeta Delgado}

2009. El enigmático mundo de las pipas coloniales. En Mar desnudo. Revista cubana de arte y literatura. No. 17.

\section{Langiano, M. del C.}

2015. Documentos y registro arqueológico en la sociedad de frontera. La pampa boanerense entre 1850 y 18980 thttp://www.incuapa-conicet.gob.ar/wp-content/uploads/Dra-Maria-del-Carmen-Langiano2015.pdf

\section{Leclaire A.}

2008. Repertories des marques. Pipiers St. Quentiniois. XVIII- XIX siècles. M. Leclaire ed. Paris.

\section{Leoni, J., D.S. Tamburini, T. Acedo de Reinoso y G. Scarafia}

2007. De balas perdidas y vidrios rotos: distribución espacial de artefactos en el Fuerte General Paz (1869-1876). Revista de Arqueología Histórica Argentina y Latino Americana. № 1: 29-64

\section{Mugueta, M. A.}

2011. Una Historia de Frontera: El Cantón Tapalqué. Ediciones de La Victoria. Azul.

2013, Guardapolvos y cucharín. Una experiencia de participación popular. En Antropología Social hoy: a 10 años del nuevo siglo. Ludmila Adad y Alicia Villafañe (Coords.), Carolina Ferrer (Ed.). Universidad Nacional del Centro de la Provincia de Buenos Aires, E Book

\section{Pezzimenti, H. L.}

2002. Las primeras tarjetas postales enel siglo XIX. Primera parte. Ediciones Rememorando. Buenos Aires.

\section{Rapoport, M.}

1988. De Pellegrini a Martínez de Hoz. El modelo liberal. CEAL, Buenos Aires.

\section{Rodino, H, J.}

2003. Ego Documentos e Identidad Bonaerense. Archivo Histórico de la Provincia de Buenos Aires "Dr. Ricardo Levene”. La Plata.

Schávelzon, D. 
1991. Arqueología Histórica de Buenos Aires. La cultura material porteña de los siglos XVIII y XIX. Ed. Corregidor. Buenos Aires. Pp:142-145.

-2003. Buenos Aires negra. Arqueología de una ciudad silenciada. Emecé. Buenos Aires.

\section{Volpe S.}

2001. Tabaco y bebidas. Colección de pipas de caolín y botellas de cerveza. Rosario 1870 1890. Versión online, Rosario.

2001. Tabaco y bebidas. Colección de pipas de caolín y botellas de ce

\section{Walker, I.}

1976. Alternative uses for clay tobacco pipes and tobacco pipes fragments: some notes. Historical Archeology. Vol 10: 124-127.

\section{Zorzi, F. y P. Davey}

2011. Descripción del conjunto de pipas halladas en el sitio Bolívar 372. En Temas y problemas de la Arqueología Histórica. Editado por Mariano Ramos; Alicia Tapia; Fabián Bognanni; Mabel Fernández; Verónica Helfer; Carlos Landa; Matilde Lanza; Emmanuel Montanari; Eugenia Néspolo y Virginia Pineau. Tomo II: 203 213. Programa de Arqueología Histórica y Estudios Pluridisciplinarios (PROARHEP). Departamento de Ciencias Sociales. Universidad Nacional de Luján. Luján.

\section{DOCUMENTOS INEDITOS}

Documentos de Frontera 1878-1890 Archivo del Juzgado de Paz de Saladillo (AJPS).

Memorias de Maria Martrin Donos Camus 1885 Ms.

Recibido: 7 de mayo de 2019

Aceptado: 30 de junio de 2019 